\title{
Influence of the Particle Size on the Optical Properties of CdSe Nanoparticles
}

\author{
Preeti Gupta and M. Ramrakhiani*
}

Department Of Post Graduate Studies and Research in Physics and Electronics Rani Durgavati University Jabalpur482001 (M.P.), India

\begin{abstract}
Monodispersed CdSe nanoparticles with sufficient luminescence intensity have been prepared by chemical method in the presence of mercaptoacetic acid as a capping agent. Cadmium acetate $\left(\mathrm{Cd}(\mathrm{Ac})_{2} \cdot 2 \mathrm{H}_{2} \mathrm{O}\right)$ and sodium selenosulfate $\left(\mathrm{Na}_{2} \mathrm{SeSO}_{3}\right)$ are used as a cadmium and selenium sources respectively. The resulting nanocrystallites have been characterized by X-Ray Diffraction, UV-Vis absorption spectra and photoluminescence (PL) spectroscopy. The particle size was estimated from broadening of XRD peak using Scherrer's formula and also from effective band gap by absorption peak using effective mass approximation model. The size obtained from XRD and absorption peaks are in agreement with each other. It is observed that the samples consist of separated, well-defined spherical particles and show a small size distribution as well as a characteristic blue shift due to quantum confinement in their optical absorption as well as PL spectra. It is found that the size of CdSe nanoparticles decreases, as the concentration of capping agent is increased. The intensity of PL peak increases and the peak shift towards blue region as the particle size is reduced. The sharp PL spectra also indicate that the nanoparticles are monodispersed.
\end{abstract}

Keywords: CdSe, Nanoparticles, X-Ray Diffraction, Optical absorption, Photoluminescence.

\section{INTRODUCTION}

Semiconductor nanoparticles are currently being extensively studied in the context of their size dependent photophysical and photochemical properties. The sizes of the nanocrystals in all three dimensions are less than the debroglie wavelength of the electronic subsystem excitations. Therefore, many properties of these materials can be systematically described and understood in term of quantum confinement effects. From the viewpoint of basic science, a nanocrystal is in an intermediate state of matter between molecule-like cluster and bulk crystals, and therefore offers a possibility to trace an evolution of electronic and optical properties of the matter from atomic cluster to bulk solids. In the context of applications, nanocrystals can be efficiently used in novel light emitting devices, optical transformers, photonic switches, nanoelectronic circuitry and other applications [1]. High quality CdSe nanocrystals with uniform monodisperse size and shape are receiving much attention from the industry for the biological labeling reagents. As the size of CdSe particles decreases from $10 \mathrm{~nm}$ to $1 \mathrm{~nm}$, the percentage of surface atoms increases from 20 to $100 \%$. The surface atoms usually have unsaturated bonds or dangling bonds. These atoms have extra free energy and are more active than those in the bulk [2]. A number of techniques like sol-gel electrostatic deposition, solvent growth, DC magnetron, sputtering and chemical bath deposition have been used for the preparation of CdSe nanoparticles. There is need to

*Address correspondence to this author at the Department Of Post Graduate Studies and Research in Physics and Electronics Rani Durgavati University Jabalpur-482001 (M.P.), India; Tel: +91-761-2620933;

E-mail: mramrakhiani@hotmail.com understand how the optical and electroronic properties change with size of nanoparticles of CdSe. The present paper reports the influence of particle variation on the optoelectronic properties of CdSe nanoparticles. The CdSe nanoparticles were prepared by chemical method using mercaptoacetic acid as a capping agent. Results of XRD, absorption spectra and photoluminescence emission spectra are presented and discussed.

\section{EXPERIMENTAL}

\subsection{Preparation of samples}

Nanocystals of CdSe were prepared by chemical method described by Zhang et al. [3]. All the chemicals used were of analytical grade. Cadmium acetate $\left(\mathrm{Cd}(\mathrm{Ac})_{2} \cdot 2 \mathrm{H}_{2} \mathrm{O}\right)$ was used as the cadmium source and sodium selenosulfate $\left(\mathrm{Na}_{2} \mathrm{SeSO}_{3}\right)$ was chosen as the selenium source. Aqueous solution of cadmium acetate $(2 \mathrm{mmol})$ and mercaptoacetic acid $\left(\mathrm{HSCH}_{2} \mathrm{COOH}\right)$ was prepared and its $\mathrm{pH}$ was adjusted as 10 by adding $1 \mathrm{M}$ solution of $\mathrm{NaOH}$. The solution was deaeraced with $\mathrm{N}_{2}$ bubbling for 30 minutes. The solution of sodium selenosulfate $\left(\mathrm{Na}_{2} \mathrm{SeSO}_{3}\right)$ was prepared freshly by dissolving Se powder $(0.02 \mathrm{~mol})$ in $\mathrm{Na}_{2} \mathrm{SO}_{3}$ solution $(0.2$ mol) and mixed to cadmium acetate solution. After this, 2 propanol was added drop-wise while stirring the mixture till it becomes turbid. The precipitate was separated through centrifugation to get dry powder. Samples have been prepared by taking different concentration of mercaptoacetic acid as:

$\mathrm{C} 1=6 \mathrm{mmol}, \quad \mathrm{C} 2=7 \mathrm{mmol}, \quad \mathrm{C} 3=8 \mathrm{mmol}, \quad \mathrm{C} 4=9 \mathrm{mmol}$ and C5 $=10 \mathrm{mmol}$. 


\subsection{Characterization of Samples}

The CdSe nanocrystalline samples were characterized by $\mathrm{X}$-ray diffraction for their crystal structure and particle size. X-ray diffraction (XRD) was taken on a Rigaku Rotating Anode (H-3R) diffractometer operating in the Bragg configuration using $\mathrm{Cu} \mathrm{K \alpha}$ radiation. The accelerating voltage was set at $40 \mathrm{KV}$ with a 100 milliamp flux.

The optical properties of CdSe nanocrystallites were studied through optical absorption and photoluminescence measurements. Room temperature UV-vis absorption spectra were obtained with a Perkin Elmer $\lambda-12$ spectrometer. The size of the CdSe nanocrystallites was estimated from blue shift in absorption peak.

Photoluminescence measurements were performed at room temperature using UV lamp and $300 \mathrm{~nm}$ filter. The intensity of emitted light at different wavelength was measured using the HM-104 monochromator and RCA-931 photomultiplier tube.

\section{RESULTS AND DISCUSSION}

\subsection{X-Ray Diffraction}

The X-ray diffraction studies were carried out for the powder samples and typical diffractograms for the five samples C1, C2, C3, C4 and C5 are shown in Fig (1). The diffraction peaks indicate the nanocrystalline nature. These peaks at angles $(2 \theta)$ of 25.391, 41.999, 45.810, 48.888, $49.718,50.699$ and 52.150 correspond to the reflection from: (002), (110), (103), (200), (112), (201) and (004) crystal planes, respectively (JCPDS card no.-060630). The XRD pattern is identical to the hexagonal phase with Wurtzite structure with space group $\mathrm{P}_{3 \mathrm{mc}}(186)$ and unit cell parameter $\mathrm{a}=\mathrm{b}=4.299 \AA$ and $\mathrm{c}=7.010 \AA$. In these samples (002) plane is very clear and abundant which indicates preferential growth of crystallites in this particular direction. The average crystal sizes (d) were calculated based on the width of the peak due to (002) planes by using the Scherrer's formula [4]:

$\mathrm{d}=0.94 \lambda / \mathrm{B} \cos \theta$

where $\lambda$ is the wavelength of X-ray used, B is the full width at half maximum and $\theta$ is the Bragg's angle of reflection. The average crystalline size (Table 1) calculated from eq (1) implies that the growth of the CdSe particles can be controlled by the mercaptoacetic acid (MAA) molecules.

\subsection{Absorption Spectroscopy}

Fig (2) shows the absorption spectra of the CdSe nanoparticles synthesized with the different concentration of mercaptoacetic acid increasing from 6 through 7, 8, 9 to $10 \mathrm{mmol}$. The first peak in the absorption spectrum shift to lower wavelength from 350 through $345,340,330$ to 310 $\mathrm{nm}$, The optical properties are strongly dependent on the particle size. It can be seen from the spectra that there is practically uniform absorption in the visible range (790-390 $\mathrm{nm})$. Absorption increases suddenly in the UV region. In addition, the absorbance between 490 to $790 \mathrm{~nm}$ increases with increasing capping agent concentration, which further indicates that the higher the capping agent concentration, the smaller the size of CdSe nanoparticles obtained.

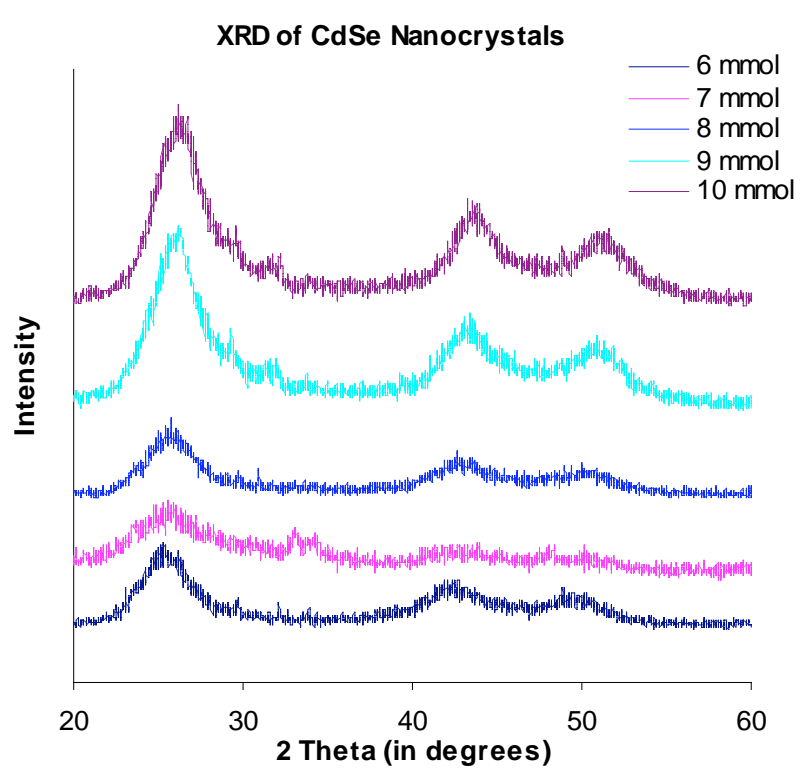

Fig. (1). X-Ray diffractograms of CdSe nanoparticles for various Concentrations of capping agent.

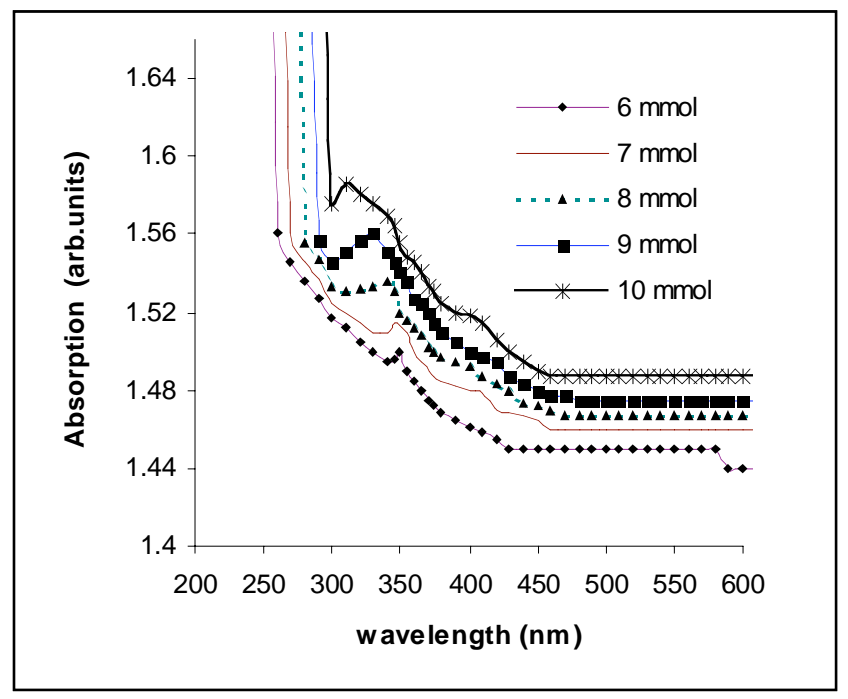

Fig. (2). Absorption spectra CdSe nanoparticles for various Concentrations of capping agent.

The most direct way of extracting the optical band gap is to simply determine the photon energy at which there is sudden increase in the absorption. For bulk samples, the band gap is estimated from the $(\alpha h v)^{2}$ vs hv plot, where $\alpha$ is the absorption coefficient and hv is the photon energy. But for nanocrystalline samples the band gap is determined from absorption maxima. The optical band gap of the nanocrystalline samples are calculated from the absorption peak using the formula

$\mathrm{E}_{\mathrm{g}}=\mathrm{hc} / \lambda$

where $\mathrm{h}$ is the Plank's constant, $\mathrm{c}$ is the velocity of light and $\lambda$ is the wavelength at which, absorption peak is obtained.

The dependence of the energy of electron transitions between quantized levels of the valance and conduction bands on the particle size was used to estimate the particle size. 
Such transition is often called excitonic [5], because an electron-hole pair generated by light absorption is similar to the Wannier-Mott exciton in a bulk crystal. In this paper, attention is paid to the particles with average sizes $d=2.5 \ldots 2.8$ $\mathrm{nm}$, for which the excitonic Bohr radius $\left(\mathrm{a}_{\mathrm{B}}=5.4 \mathrm{~nm}\right)$ [6] exceeds a particle size. That is why for these particles a strong confinement is realized.

The simplest model based on the effective-mass approximation predicts the effective band gap energy due to three- dimensional confinement comparing to the bulk one as [7] peak is obtained at photon energies less than the effective band observed by absorption peak and the peak shifts towards higher energy with reducing particle size. The results may be explained as follows.

The photons of higher energy $(4.1 \mathrm{eV})$ excite electrons from valance band (VB) to conduction band (CB) by vertical transitions and within $10^{-11} \mathrm{sec}$ the electrons comes to the bottom of CB dissipating the excess energy as lattice vibrations. Then the electrons drop to the VB and recombine with hole emitting the photons of lower energy as the FranckConden principle. The absorption peak corresponds to verti-

$$
\mathrm{E}_{\mathrm{g}}^{\prime}=\mathrm{E}_{\mathrm{g}}+\frac{\hbar^{2} \pi^{2}}{2 \mathrm{r}^{2}}\left[\frac{1}{\mathrm{~m}_{\mathrm{e}}^{*}}+\frac{1}{\mathrm{~m}_{\mathrm{h}}^{*}}\right]-\frac{1.786 \mathrm{e}^{2}}{\varepsilon \mathrm{r}}-\frac{0.124 \mathrm{e}^{4}}{\mathrm{~h}^{2} \varepsilon^{2}}\left[\frac{1}{\mathrm{~m}_{\mathrm{e}}^{*}}+\frac{1}{\mathrm{~m}_{\mathrm{h}}^{*}}\right]^{-1}
$$

where Eg is the band gap of bulk semiconductor, $\mathrm{r}$ is the radius of the particle, $\mathrm{m}_{\mathrm{e}}{ }^{*}$ is the electron effective mass, $\mathrm{m}_{\mathrm{h}}{ }^{*}$ is the hole effective masses, $\varepsilon$ is the dielectric constant of semiconductor and e the electric charge. The second term in the eq (3) represents the kinetic energy, the third term represents the Coulomb energy, and the last term is a result of the correlation effect. In general the kinetic energy is dominant and the last two terms are much smaller. Therefore in many cases, these two may be neglected. The band gap values of the particles formed with various concentration of the capping agent and the particle sizes estimated using the eq (3) are given in the Table 1. It is clear that the particle size is reduced as the capping agent concentration is increased. cal upward transition from the top of VB where as emission peak corresponds to vertical downward transition from lowest level of CB.

The luminescence intensity of nanocrystallite samples produced using $300 \mathrm{~nm}$ excitation wavelength becomes stronger with increasing concentration of mercaptoacetic acid (MAA). It is expected that the more amount of surface capping agent lead to the complete capping of CdSe surface and enhancement of band edge emission. Adding more MAA resulted in a shift of the luminescence emission to shorter wavelength and broader peak. When the particle size decreases, the effective band gap increases, therefore, the emitted photon has comparatively higher energy giving pho-

Table 1. Band Gap Values and Particle Sizes of CdSe Nanoparticles

\begin{tabular}{|c|c|c|c|c|c|}
\hline Sample & Concentration (in mmol) & $\begin{array}{c}\text { Particle size by XRD) } \\
\text { (in nm) }\end{array}$ & $\begin{array}{c}\text { Absorption Peak } \\
\text { (in nm) }\end{array}$ & $\begin{array}{c}\text { Band Gap Eg } \\
\text { (In eV) }\end{array}$ & $\begin{array}{c}\text { Diameter by EMA } \\
\text { (In nm) }\end{array}$ \\
\hline \hline C1 & 6 & 2.97 & 350 & 3.54 & 2.86 \\
\hline C2 & 7 & 2.94 & 345 & 3.60 & 2.82 \\
\hline C3 & 8 & 2.77 & 340 & 3.75 & 2.79 \\
\hline C4 & 9 & 2.76 & 330 & 4 & 2.71 \\
\hline C5 & 10 & 2.58 & 310 & 2.53 \\
\hline
\end{tabular}

It is seen from the Table $\mathbf{1}$ that the sizes calculated from broadening of XRD peak by using eq (1) were found to agree fairly with those estimated from the absorption peaks by using eq (3). The variation of effective band gap and particle size by changing capping agent concentration is shown in Fig (3). It can be observed that for concentration higher than $9 \mathrm{mmol}$, there is sudden increase in the effective band gap of the CdSe nanocrystals indicating small crystal size.

\subsection{PL Spectroscopy}

The PL spectra of the nanocrystalline CdSe samples are shown in Fig. (4). A single peak was obtained at $365 \mathrm{~nm}$, $360 \mathrm{~nm}, 355 \mathrm{~nm}, 350 \mathrm{~nm}$ and $345 \mathrm{~nm}$ for C1, C2, C3, C4 and $\mathrm{C} 5$ samples respectively. It can be noted that the PL toluminescence peak at shorter wavelength. The full width at half maxima (FWHM) is $39.60 \mathrm{~nm}, 45.26 \mathrm{~nm}, 47.15 \mathrm{~nm}$, $60.35 \mathrm{~nm}$ and $66.01 \mathrm{~nm}$ for $\mathrm{C} 1, \mathrm{C} 2, \mathrm{C} 3, \mathrm{C} 4$ and C5 concentration respectively. The FWHM increases as the capping agent concentration increases. The size distribution of the quantum dots is what leads to a range of individual emissions that the fluorimeter collects and display as a gaussion distribution (inhomogeneous broadening). The increase in FWHM of PL peak with increasing capping agent, indicating that for higher capping agent concentration, though there is more number of particles with smaller size, the range of sizes is also increased, giving a broader PL peak.

There are many reports of absorption and emission of CdSe nanocrystals prepared by different techniques. Sondi $e t$ 


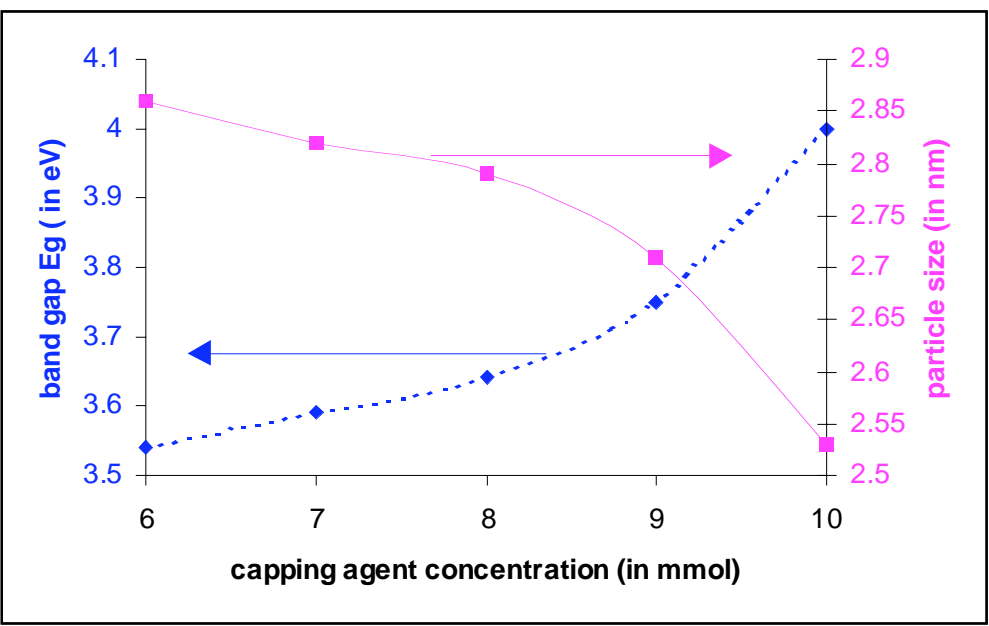

Fig. (3). Variation of effective band gap and particle size with capping agent concentration.

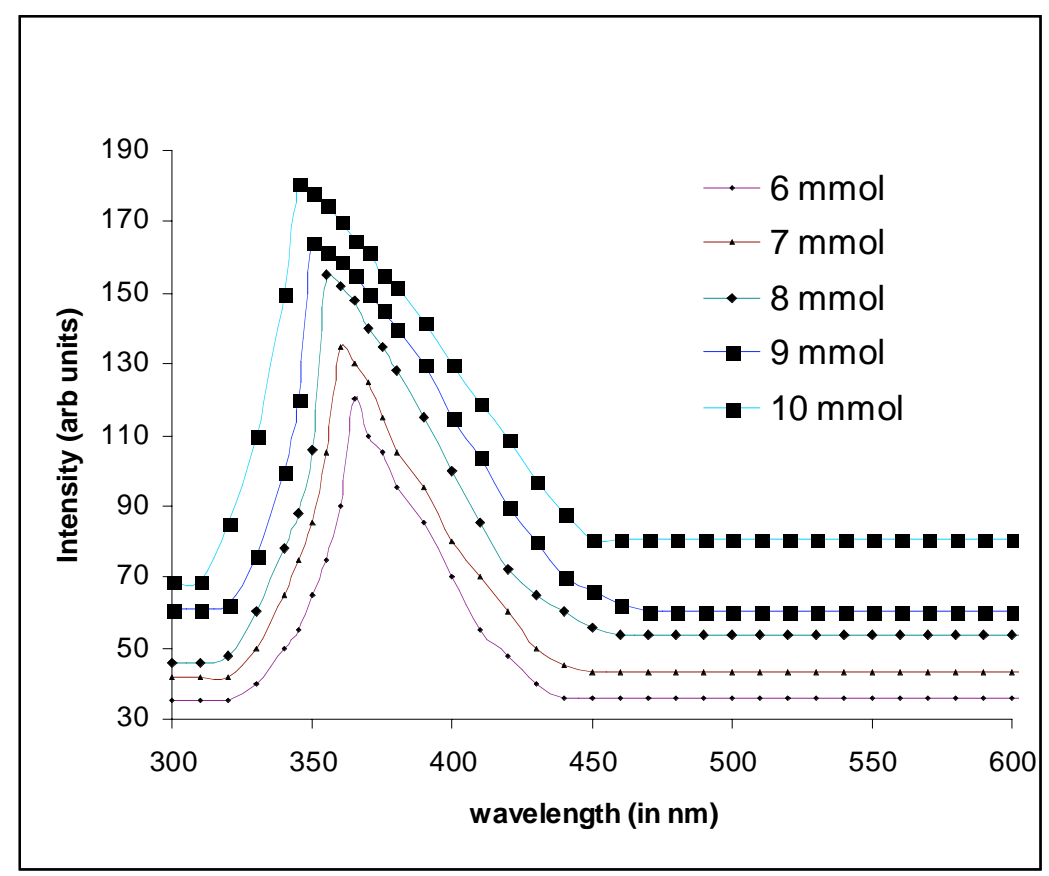

Fig. (4). Photoluminescence spectra of CdSe nanoparticles for various concentrations of capping agent.

al. [8] prepared highly luminescent CdSe particles in organic media by using aminodextron as a capping agent with excitation wavelength of $380 \mathrm{~nm}$. The absorption and photoluminescence spectra were blue shifted in agreement of our results. They also obtain band edge emission. Murali et al. [9] have prepared CdSe films from different duty cycle by pulse plating method and reported their photoluminescence. The emission spectra peaks at $520 \mathrm{~nm}$ for the films deposited at $50 \%$ duty cycle. This peak is observed to shift towards blue region $(410 \mathrm{~nm})$ as the duty cycle decreases to $6 \%$. This may be because of the increase in band gap due to decrease in particle size. Particle sizes range from $12.8 \mathrm{~nm}$ to $50.3 \mathrm{~nm}$, which are quite larger than those obtain in present work. Boatman et al. [10] have reported the absorption and emission spectra of CdSe quantum dots with particle sizes [11] ranging from approximately $1.8 \mathrm{~nm}$ to $4.00 \mathrm{~nm}$. The emis- sion wavelength is obtained ranging from $490 \mathrm{~nm}$ to $580 \mathrm{~nm}$ by excitation from $400 \mathrm{~nm}$. Shravas.S.Ashtaputre et al. [12] synthesized strongly luminescent CdSe nanoparticles by chemical routes using 2 mercaptoethanol as a capping agent. Absorption peaks were obtained at $360 \mathrm{~nm}$ and $385 \mathrm{~nm}$ for $0.01 \mathrm{M}$ (sample a) \&0.1 M (sample b) concentrations respectively. These samples show strong blue shift in absorption spectra. The photoluminescence spectra at excitation wavelength $362 \mathrm{~nm}$ exhibits a sharp peak at $430 \mathrm{~nm}$ and weak peak at $\sim 414 \mathrm{~nm}$ (sample a). In case of sample b, showing red luminescence, the excitation wavelength was $\lambda_{\text {ex }}=371$ $\mathrm{nm}$ and emission spectrum exhibits broad peak at $581 \mathrm{~nm}$ which may be due to the formation of defect levels along with peak at $437 \mathrm{~nm}$. The second peak is not observed in present studies showing a good capping and negligible surface/ defects states. 


\section{CONCLUSION}

The following conclusions can be drawn from the results of the studies on the effect of the size of CdSe nanoparticles.

1. CdSe nanocrystals can be synthesized by chemical capping method with narrow size distribution.

2. The average particle size is reduced by increasing capping agent concentration.

3. In absorption spectrum, peak is obtained in UV region and shifts towards lower wavelength by increasing the capping agent concentration. This indicates increase in effective band gap

4. PL spectrum excited by $300 \mathrm{~nm}$ shows a Stoke shifted peak in UV region. This peak also shifts towards lower side as the nanocrystal size is reduced.

5. For smaller average size, there is larger distribution of nanocrystalline sizes, causing broadening of PL peak.

\section{ACKNOWLEDGMENT}

The authors gratefully acknowledge the help provided by IUC-DAE Indore, India for XRD studies at this center.

\section{REFERENCES}

[1] Kapitonov, A.M.; Stupak, A.P.; Gaponenko, S.V.; Petrov, E.P.; Ragach, A.L.; Eychmiiller, A. Luminescence properties of thiol stabilized CdTe nanocrystals. J. Phys. Chem. B, 1999, 103, 10109.
[2] Wang, Y.; Herron, N. Optical properties of cadmium sulfide and lead II sulfide clusters encapsulated in zeolites. J. Phys. Chem., 1987, 91, 257.

[3] Zhang, S.; Yu, J.; Li, X.; Tian, W. Photoluminescence properties of mercaptocarboxylic acid stabilized CdSe nanoparticles covered with polyelectrolyte. Nanotechnology, 2004, 15, 1108.

[4] Pattabi, M.; Saraswathi A. B. Effect of precursor concentration on particle size of mercaptopropionic acetic acid capped CdS nanoparticles. J. N. Mater. Eleectrochem. Syst., 2007, 10, 43.

[5] Chestnoy, N.; Harris, T.D.; Hull, R.; Brus, L.E. Luminescence and photophysics of CdS semiconductor clusters: The nature of the emitting electronic state. J. Phys. Chem., 1986, 90, 3393.

[6] Lifshitz, E; Dag, I.; Litvin, I.; Hodes, G. Gorer, S.; Reisfeld, R. Zelner, M.; Minti, H. Optical properties of CdSe nanoaparticle film prepared by chemical deposition and sol-gel method. Chem. Phys. Lett., 1988, 288, 188.

[7] Kayanuma, Y. Quantum -size effects of interfacing electrons and holes in semiconductor microcrystals with spherical shape. Phys. Rev. B, 1988, 38 (14), 9797.

[8] Sondi, I.; Siiman, O.; Matijevic, E. Synthesis of CdSe nanoparticles in the presence of aminodextran as stabilizing and capping agent. $J$. Colloid Interface Sci., 2004, 275, 503.

[9] Murali, K.R.; Swaminathan, V.; Trivedi, D. C. Characteristics of nanocrystalline CdSe films. Solar Energy Mater. Solar Cells, 2004, 81(1), 113.

[10] Boatman, E. M.; Lisensky, G. C.; Nordell, K. J. A safer, easier and faster synthesis for CdSe quantum dots nanocrystals. J. Chem. Educ., 2005, 82(11), 1697.

[11] Murray, C. B.; Norris, D. J.; Bawendi, M. G. Synthesis and characterization of nearly monodispersed $\mathrm{CdE}(\mathrm{E}=\mathrm{S}, \mathrm{Se}, \mathrm{Te})$ semiconductor nanocrystallites. J. Am. Chem. Soc., 1993, 115, 8706.

[12] Ashtapurte, S. S.; Deshpande, A.; Marathe, S.; Wankhede, M.E.; Chimanpure, J.; Pasricha, R.; Urban, J.; Haram, S.K.; Gosavi, S.W.; Kulkarni, S.K. Synthesis and analysis of $\mathrm{ZnO}$ and CdSe nanoparticles. J. Phys., 2005, 65(4), 615. 